\section{Should Direct Thrombin Inhibitors Replace Warfarin for Prophylaxis of Thromboembolism in Canadians with Atrial Fibrillation?}

\section{THE "PRO" SIDE}

Warfarin is an effective agent for thromboprophylaxis in patients with atrial fibrillation. ${ }^{1}$ Unfortunately, however, this drug also has many limitations: a narrow therapeutic index, multiple drug and food interactions, and genetic variability in its metabolism, to name a few. These limitations contribute to the challenge of optimal use of warfarin, with the result that warfarin is not prescribed frequently enough, nor is it prescribed effectively. For warfarin to be effective, the patient's international normalized ratio (INR) must be within the therapeutic range at least $60 \%$ of the time. ${ }^{2}$ This requirement is frequently not achieved, with devastating consequences. Gladstone and others ${ }^{3}$ reviewed the Registry of the Canadian Stroke Network (now the Ontario Stroke Registry) to identify patients admitted to hospital with acute ischemic stroke who had a known history of atrial fibrillation and who were at high risk of stroke. The study reviewed data for 597 patients with indications for anticoagulation and no contraindications. The strokes experienced by these patients were disabling in $60 \%$ of cases and fatal in $20 \% .^{3}$ Only $40 \%$ of the patients had been receiving warfarin, with $31 \%$ receiving antiplatelet agents and 29\% receiving no antithrombotics at all. Of those who were receiving warfarin, the majority (74\%) had a subtherapeutic INR. Underutilization of warfarin has been reported by other authors. For example, Bungard and others ${ }^{4}$ found that the reported rates of warfarin use for patients without contraindications ranged from $15 \%$ to $79 \%$, with the majority of trials reporting between $20 \%$ and $40 \%$ of patients having a prescription for warfarin. The reasons for underuse are multiple, from patients' unwillingness to take the drug to physicians' reluctance to prescribe it because of the complexity of dosing, the need for regular monitoring, and inadequate remuneration.

Given the multiple challenges associated with warfarin, and the resultant underuse and less-than-optimal management of warfarin therapy, it is important to identify and use alternatives that are not subject to these challenges. The new oral anticoagulants meet these criteria, being effective, safe, and more convenient than warfarin.

The efficacy of 3 new agents - dabigatran, rivaroxaban, and apixaban — has been demonstrated in the RE-LY, ROCK-
ET AF, ${ }^{6}$ and ARISTOTLE ${ }^{7}$ trials, respectively. The ROCKET $\mathrm{AF}$ and ARISTOTLE trials were randomized, double-blind trials, whereas the RE-LY trial used a prospective, randomized, open-label, blinded end point design in which the 2 doses of dabigatran were blinded and warfarin was administered in an open-label fashion. Each agent was shown to be non-inferior to warfarin, ${ }^{5-7}$ and in the cases of dabigatran $150 \mathrm{mg} \mathrm{bid}$ and apixaban, ${ }^{7}$ the agents were superior in terms of reducing the risk of stroke or systemic embolism (the primary outcome of these trials). In addition, the use of apixaban was associated with a lower annual mortality rate than warfarin; $3.52 \%$ versus $3.94 \%$ (hazard ratio 0.89 , 95\% confidence interval 0.80-0.998;

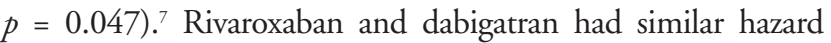
ratios for mortality ( 0.85 and 0.88 , respectively), although they did not reach statistical significance., ${ }^{5,6}$ These trials have been criticized because INR control was poor and not reflective of current practice; furthermore, when INR control was good (time in therapeutic range [TTR] $>65.5 \%$ ), the superiority of these agents disappeared. ${ }^{8}$ However, the reality is that good TTR is typically not achieved in practice. Van Walraven and others ${ }^{9}$ found that the overall rate was $63.6 \%$, but when the data were broken down by care setting, the TTR for community practice was only $56.7 \%$, and for the majority of patients receiving warfarin, care is managed by community practitioners. Recently, the Canadian Agency for Drugs and Technology in Health (CADTH) reviewed how warfarin management could be optimized ${ }^{10}$ and recommended that the drug be managed by a well-coordinated, structured approach dedicated to anticoagulation therapy. What is not known is the percentage of the Canadian population that does or could access such care.

The major concern with any anticoagulant is bleeding. Apixaban had lower rates of major bleeding than warfarin. ${ }^{\text {? }}$ Although dabigatran $150 \mathrm{mg}$ bid was associated with a higher rate of gastrointestinal bleeding than warfarin, the overall rate of major bleeding did not differ between the 2 agents, and the 110 -mg bid dose of dabigatran was associated with lower rates of bleeding than warfarin. ${ }^{5}$ Rivaroxaban had rates of major bleeding similar to those for warfarin. ${ }^{6}$ All 3 agents displayed a lower annual rate of intracranial hemorrhage than warfarin $(0.3 \%-0.5 \%$ versus $0.7 \%-0.8 \%)$, which was independent of the degree of INR control. ${ }^{10}$ Concern about intracranial hemorrhage is one of the most frequently quoted reasons for not prescribing warfarin, and it is very encouraging that all of these agents had lower rates of this adverse effect.

Several features make the new oral anticoagulation agents more convenient than warfarin. First, they have a rapid onset, with the anticoagulant effect becoming apparent within $2-4 \mathrm{~h}$ after the first dose, rather than the 4-5 days required for warfarin. These agents have been heavily promoted as not 
requiring any monitoring, and for the most part this is true. In all 3 studies, the efficacy and safety results summarized above were achieved with a fixed-dose, unmonitored regimen. What has become apparent in the postmarketing period is that it is not anticoagulant status, but rather the patient's renal function that requires ongoing monitoring, to ensure that the drug is still an appropriate choice and the dose is correct. ${ }^{11}$ Although routine anticoagulation monitoring is not required, monitoring can be performed with these agents. A modified thrombin time assay (Hemoclot [Aniara]) correlates with dabigatran concentration, and any elevation in the thrombin time or activated partial thromboplastin time reflects the presence of dabigatran. ${ }^{12}$ Rivaroxaban effects can be measured by rivaroxaban-calibrated anti-Xa levels. ${ }^{13}$ Apixaban's effect can be measured by means of the anti-Xa levels currently used for low-molecular-weight heparins. ${ }^{14}$ Both rivaroxaban and apixaban will elevate the prothrombin time and INR. Although routine monitoring is not required, it is beneficial to have the ability to determine the presence of drug in the periprocedure or perioperative period through currently available tests. In the future, when the therapeutic ranges have been determined, such testing will be used to tailor the dosages of these medications for better efficacy and safety.

One concern about the new oral anticoagulants, quoted by many, is the lack of an antidote or reversal agent. The effect of currently available factors, such as active and inactive prothrombin complex concentrates (e.g., factor VIII inhibitor bypassing activity, also known as FEIBA, and Octaplex [Octapharma]) or activated recombinant factor VII (also known as rVIIa), on human in vivo laboratory parameters and the ability of these agents to control or minimize bleeding are under investigation. Major and life-threatening bleeding can be managed by supportive measures. ${ }^{15}$ In fact, concern about a lack of reversal agent may also apply to warfarin, which is promoted as having antidotes available, specifically vitamin $\mathrm{K}$ and prothrombin complex concentrates. However, although these agents have been shown to correct laboratory parameters, their impact on clinical outcomes is not well established. ${ }^{16}$

Cost is always a concern with new medications. In terms of direct drug cost, the new agents are significantly more expensive than warfarin ( $\$ 3 /$ month for warfarin versus $\$ 96 /$ month for dabigatran or $\$ 85 /$ month for rivaroxaban). However, once monitoring and indirect costs are considered, the difference is not as great. The 3-month cost of warfarin therapy in 2009, from a ministry of health perspective (including costs for the drug, prothrombin time tests, and physicians and other health care providers), was estimated at $\$ 108$ to $\$ 198$, and this estimate increased to $\$ 187$ to $\$ 243$ when total societal costs, including patient and overhead costs, were considered. ${ }^{17}$ What is not known is the cost of the well-coordinated, structured approach outlined by CADTH as being essential to achieving optimal $\mathrm{TTR}^{10}$ and whether this approach would be available to all Canadians receiving warfarin.

Given the limitations of warfarin, combined with the reluctance of both patients and health care providers to use this drug, the emergence of alternative agents has been eagerly awaited. The new oral anticoagulants are at least as effective as warfarin (and often superior in efficacy), they are as safe or safer with respect to bleeding (particularly intracranial hemorrhage), and they are more convenient to use; as such, they should be considered first-line agents. Although these new agents do require some monitoring, and the choices of agent and dose require careful consideration, they represent a significant improvement over what should now be considered the secondline agent, warfarin.

\section{References}

1. BoHart RG, Pearce LA, Aguilar MI. Meta-analysis: antithrombotic therapy to prevent stroke in patients who have nonvalvular atrial fibrillation. Ann Intern Med 2007;146(12):857-867.

2. White HD, Gruber M, Feyzi J, Kaatz S, Tse HF, Husted S, et al. Comparison of outcomes among patients randomized to warfarin therapy according to anticoagulant control: results from SPORTIF III and V. Arch Intern Med 2007;167(3):239-245.

3. Gladstone DJ, Bui E, Fang J, Laupacis A, Lindsay MP, Tu JV, et al. Potentially preventable strokes in high-risk patients with atrial fibrillation who are not adequately anticoagulated. Stroke 2009;40(1):235-240.

4. Bungard TJ, Ghali WA, Teo KK, McAlister FA, Tsuyuki RT. Why do patients with atrial fibrillation not receive warfarin? Arch Intern Med 2000;160(1):41-46.

5. Connolly SJ, Ezekowitz MD, Yusuf S, Eikelboom J, Oldgren J, Parekh A, et al.; RE-LY Steering Committee and Investigators. Dabigatran versus warfarin in patients with atrial fibrillation. N Engl J Med 2009;361(12):1139-1151. Erratum in: N Engl J Med 2010;363(19):1877.

6. Patel MR, Mahaffey KW, Garg J, Pan G, Singer DE, Hacke W, et al.; ROCKET AF Investigators. Rivaroxaban versus warfarin in nonvalvular atrial fibrillation. N Engl J Med 2011;365(10):883-891.

7. Granger CB, Alexander JH, McMurray JJ, Lopes RD, Hylek EM, Hanna M, et al.; ARISTOTLE Committee and Investigators. Apixaban versus warfarin in patients with atrial fibrillation. N Engl J Med 2011;365(11):981-992.

8. Wallentin L, Yusuf S, Ezekowitz MD, Alings M, Flather M, Franzosi MG, et al.; RE-LY Investigators. Efficacy and safety of dabigatran compared with warfarin at different levels of international normalised ratio control for stroke prevention in atrial fibrillation: an analysis of the RE-LY trial. Lancet 2010;376(9745):975-983.

9. van Walraven C, Jennings A, Oake N, Fergusson D, Forster AJ. Effect of study setting on anticoagulation control: a systemic review and metaregression. Chest 2006;129(5):1155-1166.

10. Optimal warfarin management for the prevention of thromboembolic events in patients with atrial fibrillation: a systematic review of the clinical evidence. Optimal Use Report, Vol 1, Issue 2a. Ottawa (ON): Canadian Agency for Drugs and Technologies in Health; 2011 Nov [cited 2012 May 5]. Available from: http://cadth.ca/media/pdf/OP0508_warfarin-science-report-1_ clinical_e.pdf

11. Pradax (dabigatran etexilate) — updated labelling regarding kidney function assessment and use in patients with certain types of heart valve disease or artificial heart valves — for health professionals. Ottawa (ON): Health Canada; 2012 Mar 16 [cited 2012 May 5]. Available from: www.hc-sc.gc.ca/dhpmps/medeff/advisories-avis/prof/_2012/pradax_hpc-cps-eng.php

12. van Ryn J, Stangier J, Haertter S, Liesenfeld KH, Wienen W, Feuring M, et al. Dabigatran etexilate - a novel, reversible, oral direct thrombin inhibitor: interpretation of coagulation assays and reversal of anticoagulant activity. Thromb Haemost 2010;103(6):1116-1127.

13. Harenberg J, Erdle S, Marx S, Krämer R. Determination of rivaroxaban in human plasma samples. Semin Thromb Hemost 2012;38(2):178-184.

14. Barrett YC, Wang Z, Frost C, Shenker A. Clinical laboratory measurement of direct factor Xa inhibitors: anti-Xa assay is preferable to prothrombin time assay. Thromb Haemost 2010;104(6):1263-1271.

15. Kaatz S, Kouides PA, Garcia DA, Spyropolous AC, Crowther M, Douketis JD, et al. Guidance on the emergent reversal of oral thrombin and factor Xa inhibitors. Am J Hematol 2012;87 Suppl 1:S141-S145. Erratum in: Am J Hematol 2012;87(7):748.

16. Patanwala AE, Acquisto NM, Erstad BL. Prothrombin complex concentrate for critical bleeding. Ann Pharmacother 2011;45(7-8):990-999. 
17. Schulman S, Anderson DR, Bungard TJ, Jaeger T, Kahn SR, Wells P, et al. Direct and indirect costs of management of long-term warfarin therapy in Canada. J Thromb Haemost 2010;8(10):2192-200.

Heather Kertland, BScPhm, PharmD

Heart and Vascular Program

St Michael's Hospital

Leslie Dan Faculty of Pharmacy

University of Toronto

Toronto, Ontario

\section{THE "CON" SIDE}

At first glance, the new oral anticoagulants (dabigatran, rivaroxaban, and apixaban) present enticing alternatives to warfarin for clinicians, mainly for use in patients with nonvalvular atrial fibrillation. However, I feel that caution is warranted at this point, for the reasons outlined below.

Imagine making a favourable judgment about the quality of a book from its interesting cover, but then finding, once the book is opened, that some of the chapters are missing, some have not yet been written, and others can only be found in places other than the book itself. The plot of such a book would be difficult to discern. The story for each of the new oral anticoagulants is very much like this. Knowledge about each of these drugs for the indication of nonvalvular atrial fibrillation comes, in large part, from only one large phase III trial. ${ }^{1-3}$ These 3 studies represent the existing chapters of the book. However, none of the signals of harm, effects in subgroups, or claimed benefits reported in these trials has been replicated in subsequent randomized controlled trials (RCTs). Why is this lack of replication (the missing chapters of the story) a problem? Simply put, the findings of these 3 trials may be false positives (i.e., nonreplicable and not in fact "real"). ${ }^{4}$ Recent examples of falsepositive studies have been the mortality reductions observed in the first RCT of activated protein $\mathrm{C}$ in sepsis ${ }^{5}$ and in a trial of recombinant factor VIIa for treatment of acute intracerebral hemorrhage. ${ }^{6,7}$ In both of these examples, initial phase III trials found mortality reductions in favour of the new drugs, but the reductions could not be replicated in subsequent trials. ${ }^{8,9}$ Findings in the trials of new oral anticoagulants should be considered signals, in terms of both benefit and harm, that must be replicated before they can be considered definitive. More importantly, because the signals of harm reported in these studies are serious, they must be given adequate attention, and appropriate caution must be exercised, before we advocate widespread use that could potentially expose many patients to unnecessary harm. The RCTs that need to be done represent the unwritten chapters of the story.

With regard to chapters residing in places other than between the covers of the book, the current story of the new oral anticoagulants is also being told in publicly available but seldom used regulatory documents, primarily from the US Food and
Drug Administration (FDA) (www.accessdata.fda.gov/scripts/ cder/drugsatfda/) and the European Medicines Agency (www.ema.europa.eu/ema/). In particular, many signals of harm became apparent only when I and my colleagues at the Therapeutics Initiative (based at the University of British Columbia, Vancouver, British Columbia) looked into these documents. The following paragraphs, although by no means a comprehensive discussion, summarize our key concerns.

First, can the reductions in stroke being attributed to the new oral anticoagulants be believed? In particular, claims that dabigatran reduces stroke to a greater extent than warfarin should be questioned. The comparison of dabigatran against warfarin was unblinded, and the FDA medical review highlighted evidence that knowledge of treatment assignment may have affected the way in which patients were treated. ${ }^{10}$ The FDA review also pointed out that, despite blinded adjudication of outcomes, approximately $17 \%-20 \%$ of the data recorded on case report forms may have been unblinded because of the way in which investigators worded their descriptions of patients' responses. Given this potential for unblinding, the magnitude of stroke reduction reported in the initial trial ${ }^{1}$ was likely a reflection, in whole or in part, of bias and thus could easily be overturned or reduced to a clinically unimportant difference in future studies. As for hemorrhagic strokes, the rate with each of the new oral anticoagulants was lower than the rate with warfarin..$^{1-3}$ There was, however, one interesting discrepancy: if hemorrhagic strokes are reduced among patients receiving one of the new oral anticoagulants, fatal or disabling strokes should also be reduced, but such a reduction was not observed for dabigatran or rivaroxaban, ${ }^{1,2}$ relative to warfarin (and this outcome was not reported for the apixaban trial $^{3}$ ).

My second concern arises from the impression I get from media reports, marketing, and word-of-mouth in the clinical community that the new oral anticoagulants do not increase the risk of major bleeding relative to warfarin. However, for a patient or a clinician who is trying to make an informed decision about whether to consider a new oral anticoagulant, this impression is not quite accurate. In my view, it would be more accurate to say that there appears to be a bleeding trade-off. For dabigatran and rivaroxaban, rates of intracranial hemorrhage were lower, whereas rates of major gastrointestinal bleeding were higher, relative to warfarin. ${ }^{1,2}$ The apixaban RCT demonstrated absolute reductions of $1 \%$ for major bleeding and $0.7 \%$ for intracranial hemorrhage relative to warfarin (with no increase in the risk of major gastrointestinal bleeding). ${ }^{3}$ The increased risk of some types of major bleeding and decreased risk for other types may hold different levels of importance for different patients.

The third concern is that our understanding of the optimal dose for each new oral anticoagulant is still evolving. At present, monitoring of international normalized ratio (INR) is not required with these agents, and it may seem like heresy to suggest that this lack of monitoring is a "bad" thing. However, one must consider the potential riskiness of a "one- or two-dose regimen fits all" approach for these potent anticoagulants. But what do the trials tell us about the choice of dose? In fact, the story is not 
complete in the available chapters (i.e., the published RCT reports $\left.^{1-3}\right)$, and different interpretations of these data appear in the FDA reports ${ }^{10,11}$ and in independent analyses ${ }^{12}$ of the RCT data. For example, the FDA questioned the choice of rivaroxaban $20 \mathrm{mg}$ once daily, suggesting that $20 \mathrm{mg}$ twice daily might have been a better choice, given the drug's pharmacokinetics and pharmacology. ${ }^{11}$ At a higher dose, rivaroxaban might have performed better than warfarin in terms of stroke reduction, but there might also have been an increased risk of toxicity relative to what was seen with the once-daily dose. For dabigatran, others have noted that the 110-mg twice-daily dose reduced all-cause hospital admission but also increased the risk of fatal or disabling stroke relative to the 150 -mg twice-daily dose. ${ }^{12}$ In addition, the investigators in the RE-LY trial of dabigatran published a substudy which suggested that for patients older than 75 years of age, the risk of major bleeding was greater with the 150-mg twice-daily dose than with warfarin (the risk was the same for the 110-mg dose and warfarin). ${ }^{13}$ For apixaban, there are no publicly available analyses from regulators or independent groups that thoroughly explain the justification for the dose used in the ARISTOTLE trial. ${ }^{3}$

The fourth concern relates to the increased risk of thrombosis with both dabigatran and rivaroxaban. In a recently published meta-analysis, Uchino and Hernandez ${ }^{14}$ suggested a significantly increased risk of myocardial infarction or acute coronary syndrome with dabigatran relative to all comparators in RCTs for all conditions. Some have questioned the relevance of an analysis that compares dabigatran with all comparators for several different indications. The argument for such an approach is that it is has more statistical power to reveal differences because of the large patient numbers obtained by pooling data. But are these differences meaningful? The answer is yes: Uchino and Hernandez $^{14}$ found no statistical heterogeneity, which indicates that the risk of myocardial infarction or acute coronary syndrome associated with dabigatran was consistently present, despite differences among the trials. In addition, similar signals of harm were present with ximelagatran, a close relative of dabigatran. ${ }^{11}$ With rivaroxaban, the signal for rebound thrombotic risk has not been publicized. The FDA medical review ${ }^{11}$ noted that upon discontinuation of therapy in the ROCKET AF trial, ${ }^{2}$ stroke or systemic emboli occurred more frequently among patients who stopped taking rivaroxaban than among patients who stopped taking warfarin (22 versus 6 events in the 30 days after stopping). ${ }^{11}$

The final concern is based on the lack of detail about serious adverse events in all 3 trials. Comparisons of serious adverse event rates, if such events had been accounted for and reported properly $y^{15}$ by the investigators of all 3 trials, would allow an assessment of whether the signals of serious harm are outweighed by the signals of benefit. For dabigatran, our group requested details of total serious adverse events, but the manufacturer would not release the information unless we signed a confidentiality agreement. The manufacturer did tell us that strokes and deaths were not reported as serious adverse events in the published trial report, despite the fact that these occurrences are, by definition, serious adverse events. For rivaroxaban, the FDA report stated that deaths and bleeding episodes were recorded in the published data on serious adverse events, but no details have been provided as to how strokes were accounted for in the analysis of such events. ${ }^{11}$ For apixaban, no details have been reported as to how serious adverse events were counted. All of the trials reported an investigator-defined net benefit outcome, which included outcomes thought to be affected by the new oral anticoagulant but did not include all serious adverse events (i.e., events not expected to be due to these drugs); this approach has limited utility and ignores possible serious harm related to the new drugs. Without details on total serious adverse events, the true risk-benefit ratio remains unknown.

In summary, the currently available evidence for new oral anticoagulants suggests possible clinically important benefit coupled with possible clinically important serious harm. As the scientific method suggests, these initial findings should not be considered definitive evidence. Rather, they require replication in future RCTs before they can be believed. In the meantime, patients and clinicians need to be aware of all the facts that are currently available if they are to make informed decisions about these new drugs. Should patients use these drugs? Certainly they would need to be made aware of the aforementioned concerns (including the fact that a bleeding reversal agent has not been identified for any of them) before making a decision to do so. Would I take one of the new oral anticoagulants? Only if I couldn't take warfarin or acetylsalicylic acid for stroke prevention with nonvalvular atrial fibrillation. I look forward to reading subsequent chapters in the story of these new oral anticoagulants.

\section{References}

1. Connolly SJ, Ezekowitz MD, Yusuf S, Eikelboom J, Oldgren, J, Parekh A, et al.; RE-LY Steering Committee and Investigators. Dabigatran versus warfarin in patients with atrial fibrillation. $N$ Engl $J$ Med 2009;361(12):1139-1151. Erratum in: N Engl J Med 2010;363(19):1877.

2. Patel MR, Mahaffey KW, Garg J, Pan G, Singer DE, Hacke W, et al.; ROCKET AF Investigators. Rivaroxaban versus warfarin in nonvalvular atrial fibrillation. $N$ Engl J Med 2011;365(10):883-891.

3. Granger CB, Alexander JH, McMurray JJ, Lopes RD, Hylek EM, Hanna M, et al.; ARISTOTLE Committee and Investigators. Apixaban versus warfarin in patients with atrial fibrillation. N Engl J Med 2011:365(11):981-992.

4. Ioannidis JPA. Why most published research findings are false. PLoS Med 2005;2(8):e124.

5. Bernard GR, Vincent JL, Laterre PF, LaRosa SP, Dhainaut JF, LopezRodriguez A, et al.; Recombinant human protein C Worldwide Evaluation in Severe Sepsis (PROWESS) study group. Efficacy and safety of recombinant human activated protein $\mathrm{C}$ for severe sepsis. $N$ Engl $\mathrm{J}$ Med 2001;344(10):699-709.

6. Mayer SA, Brun NC, Begtrup K, Broderick J, Davis S, Diringer MN, et al.; Recombinant Activated Factor VII Intracerebral Hemorrhage Trial Investigators. Recombinant activated factor VII for acute intracerebral hemorrhage. $N$ Engl J Med 2005;352(8):777-785.

7. Mayer SA, Brun NC, Begtrup K, Broderick J, Davis S, Diringer MN, et al.; FAST Trial Investigators. Efficacy and safety of recombinant activated factor VII for acute intracerebral hemorrhage. N Engl J Med 2008;358(20): 2127-2137.

8. Martí-Carvajal AJ, Solà I, Lathyris D, Cardona AF. Human recombinant activated protein C for severe sepsis. Cochrane Database Syst Rev 2012; 3:CD004388.

9. Simpson E, Lin Y, Stanworth S, Birchall J, Doree C, Hyde C. Recombinant factor VIIa for the prevention and treatment of bleeding in patients without 
haemophilia. Cochrane Database Syst Rev 2012;3:CD005011.

10. Application number: 22-512. Medical review(s). Silver Spring (MD): Center for Drug Evaluation and Research; 2010 [cited 2012 Apr 10]. Available from: www.accessdata.fda.gov/drugsatfda_docs/nda/2010/022512Orig1s 000MedR.pdf

11. Application number: 202439Orig1s000. Medical review(s). Silver Spring (MD): Center for Drug Evaluation and Research; 2010 [cited $2012 \mathrm{Apr}$ 10]. Available from: www.accessdata.fda.gov/drugsatfda_docs/nda/2011/ 202439 Orig1s000MedR.pdf

12. Dabigatran for atrial fibrillation: why we can not rely on RE-LY. Ther Lett 2011 [cited 2012 Apr 10];80:1-2. Available from: www.ti.ubc.ca/letter80

13. Eikelboom JW, Wallentin L, Connolly SJ, Ezekowitz M, Healey JS, Oldgren J, et al. Risk of bleeding with 2 doses of dabigatran compared with warfarin in older and younger patients with atrial fibrillation an analysis of the randomized evaluation of long-term anticoagulant therapy (RE-LY) trial. Circulation 2011;123(21):2363-2372.

14. Uchino K, Hernandez A. Dabigatran association with higher risk of acute coronary events: meta-analysis of noninferiority randomized controlled trials. Arch Intern Med 2012;172(5):397-402.
15. ICH harmonised tripartite guideline. Guideline for good clinical practice E6(R1). Geneva (Switzerland): International Conference on Harmonisation of Technical Requirements for Registration of Pharmaceuticals for Human Use; 1996 Jun 10 [cited 2012 Apr 25]. Available from: www.ich.org/ fileadmin/Public_Web_Site/ICH_Products/Guidelines/Efficacy/E6_R1/ Step4/E6_R1_Guideline.pdf

\section{Aaron M Tejani, BSc(Pharm), PharmD}

Researcher

Therapeutics Initiative

University of British Columbia

Vancouver, British Columbia

\section{Acknowledgements}

I thank Vijaya Musini, Xiao-Lin Zhang, and members of the Drug Assessment Working Group of the Therapeutics Initiative for their help in interpreting the evidence regarding the new oral anticoagulants.

\section{CJHP Call for photographs}

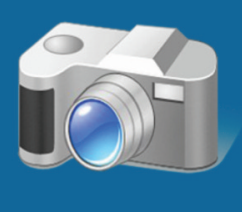

The Canadian Journal of Hospital Pharmacy is looking for photographs for the cover of the Journal. The photo theme for 2012 is:

\section{Canadian Landscapes and Scenery}

Interested participants are asked to submit a digital photo or group of photos for selection along with a short (max 150 words) write-up about the location of the photo, the date and time of the photo, and the type of equipment used. Be sure to include any stories or details that make this photograph or location unique!

Entries can be submitted to Colleen Drake, Publications Administrator, at cdrake@cshp.ca.
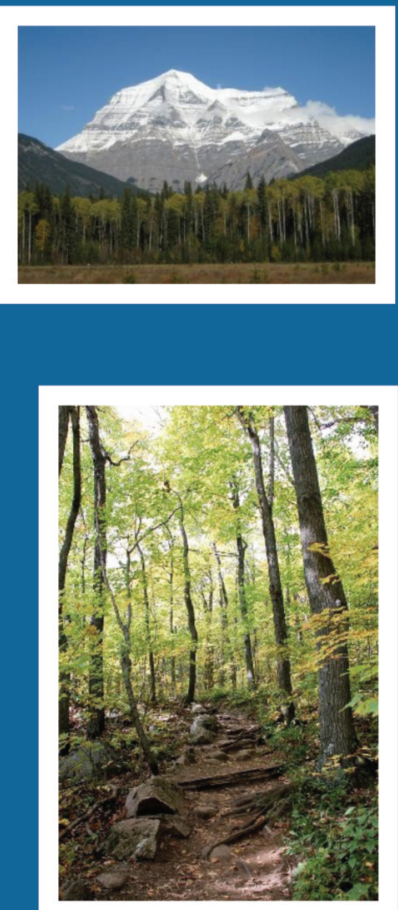